\title{
Huge thoracic tracheal tumor related to acute respiratory distress syndrome
}

\author{
Massine El Hammoumi ${ }^{1}$, Adil Zegmout ${ }^{2}$, Fouad Nya ${ }^{3}$, Mohamed Bhairis ${ }^{1}$, Marius Kamdem ${ }^{1}$, \\ El Hassane Kabiri ${ }^{1}$ \\ ${ }^{1}$ Department of Thoracic Surgery, Military Teaching Hospital Mohammed V, Rabat, Morocco \\ ${ }^{2}$ Department of Pneumology, Military Teaching Hospital Mohammed V, Rabat, Morocco \\ ${ }^{3}$ Department of Cardiac Surgery, Military Teaching Hospital Mohammed V, Rabat, Morocco
}

Kardiochirurgia i Torakochirurgia Polska 2021; 18 (3): 180-182

Adenoid cystic carcinoma of the trachea (ACCT) is an unusual low-grade tumor from the tracheal and bronchial wall salivary glands. ACCT occurs most commonly in the upper trachea, being found in the articular cartilage in the posterior part of the trachea.

We report the case of a patient with the rare condition of tracheal ACC who presented in an emergency condition that was successfully treated with a combination of surgery and radiotherapy.

Our patient was a 65-year-old, male, ex-smoker of 35 years. His condition began with mild dyspnea 2 years ago, with intense cough. With worsening of the aforementioned symptoms, he sought prompt care. In physical ex-

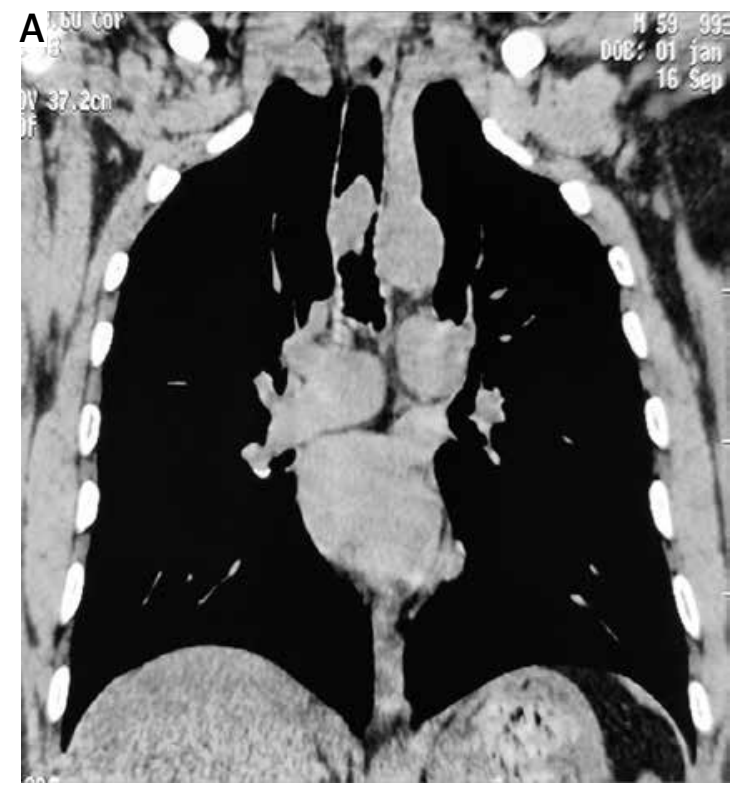

Figure 1. Chest CT with a tracheal distal tumor. A - Computed tomography $(\mathrm{CT})$ coronal view. $\mathrm{B}-\mathrm{CT}$ in transversal mediastinal view. $\mathbf{C}-\mathrm{CT}$ in transversal parenchyma view amination the blood pressure was $110 / 65 \mathrm{~mm} \mathrm{Hg}$, arterial oxygenation $\left(\mathrm{SaO}_{2}\right)$ 86\%. The probable diagnosis was a crisis of chronic obstructive pulmonary disease (COPD) or pulmonary embolism. After stabilizing the patient, injectable corticotherapy, enoxaparin and inhaled bronchodilators, a COVID-19 polymerase chain reaction (PCR) was negative. A C+ computed tomography (CT) of the chest showed an expansive lesion in the right wall of the thoracic trachea with an extension of approximately $3.1 \mathrm{~cm}$, ending at the subcarinal level, measuring $4.5 \times 2.8 \mathrm{~cm}$, with a huge stenosis of the tracheal lumen, with an exophytic component to the mediastinum (Figures $1 \mathrm{~A}-\mathrm{C}$ ). No specific COVID-19 related lesions were described. Bronchoscopy confirmed the
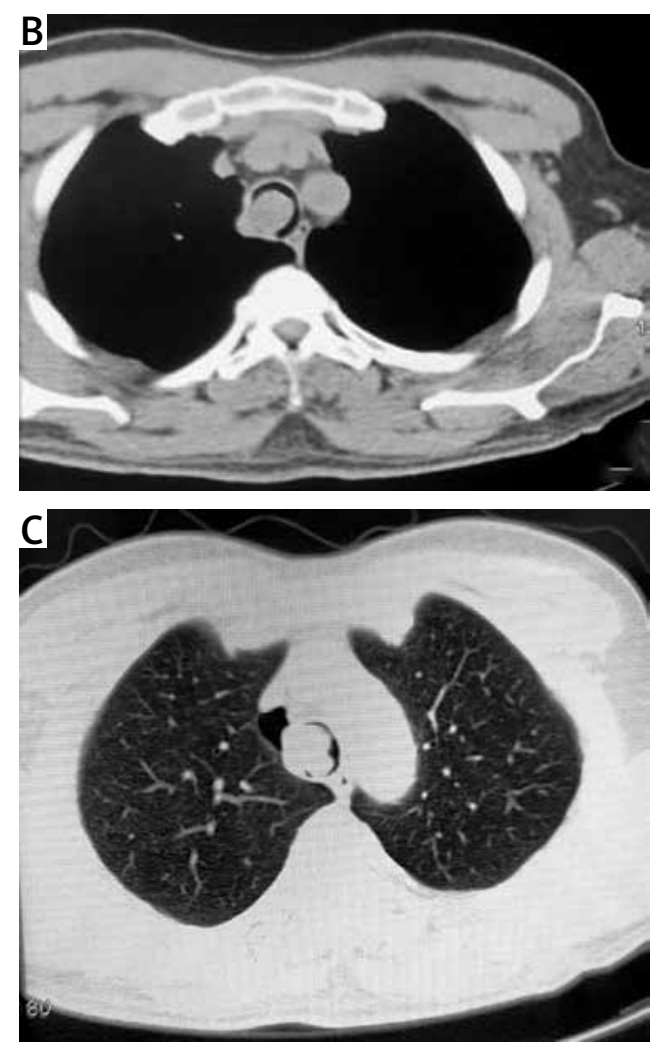

Address for correspondence: Prof. El Hammoumi Massine, Department of Thoracic Surgery, Military Teaching Hospital Mohammed V, Rabat, Morocco, e-mail: hamoumimassine@hotmail.fr

Received: 4.05.2021, accepted: 8.07.2021. 


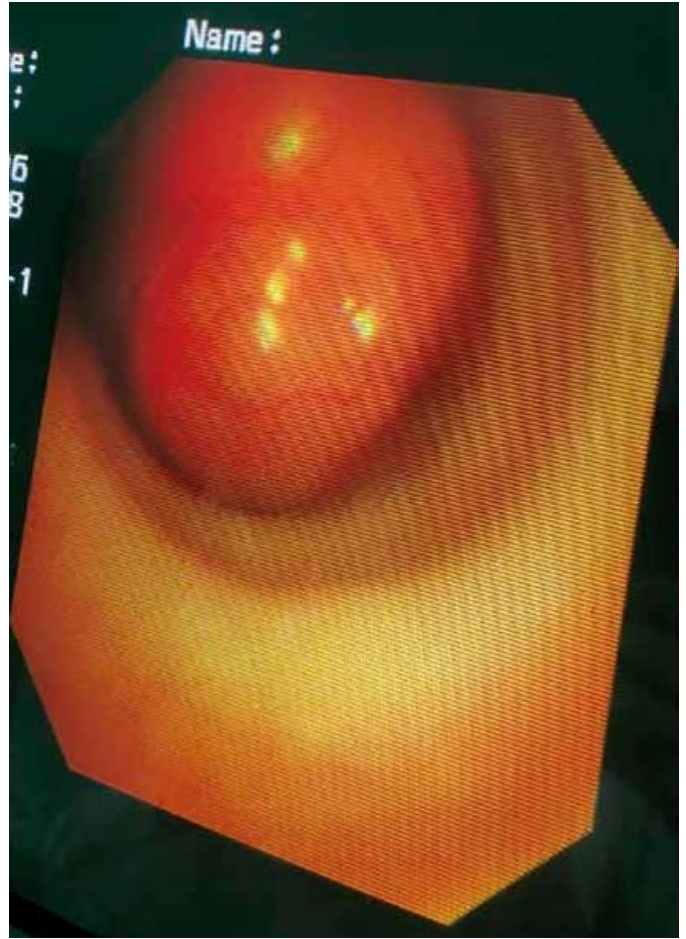

Figure 2. Endoscopic view showing a huge obstructive endoluminal tumor of the sus carinal trachea

finding of a capsulated, vascularized and friable lesion that could be manipulated in a distal portion of the trachea of $1 \mathrm{~cm}$, that of the main carina, causing significant obstruction of the airflow (Figure 2). Endotracheal biopsy of multiple fragments and histopathology confirmed an adenoid cystic carcinoma (Figure 3). The patient underwent emergency surgery 24 hours after admission: right posterolateral thoracotomy (Figure 4) under femoral ECMO with tracheal resection- distal anastomosis, one tumor block resection and interposition of an intercostal muscle flap (Figure 5) was performed. The patient remained postoperatively for 1 day in the intensive care unit (ICU). The patient recovered thoracic drainage 4 days later, and was discharged on the $5^{\text {th }}$ postoperative day. Definitive pathological examination revealed cystic adenoid carcinoma without an infiltration of adjacent soft tissues, but with perineural and angiolymphatic invasion. The patient was in outpatient follow-up, showing an absolute improvement of clinical status, with adjuvant radiotherapy being indicated. Posterior bronchoscopy was performed with good healing.

ACCT exhibits slow growth and a low rate of local and distant metastasis. It is often clinically asymptomatic and the diagnosis is based on CT. Even with good imaging results for the characterization of the primary tumor and to document lymph nodal or distant metastases, the positron emission tomography-computed tomography (PET-CT) scan is also useful for staging and has been used for treatment. A better prognosis is mainly related to the feasibility of complete resection and to the absence of metastasis. However, symptoms such as wheezing, tracheitis, hemoptysis, and stridency are often unspecific and late. Lymph node involvement has not been associated with a worse progno-

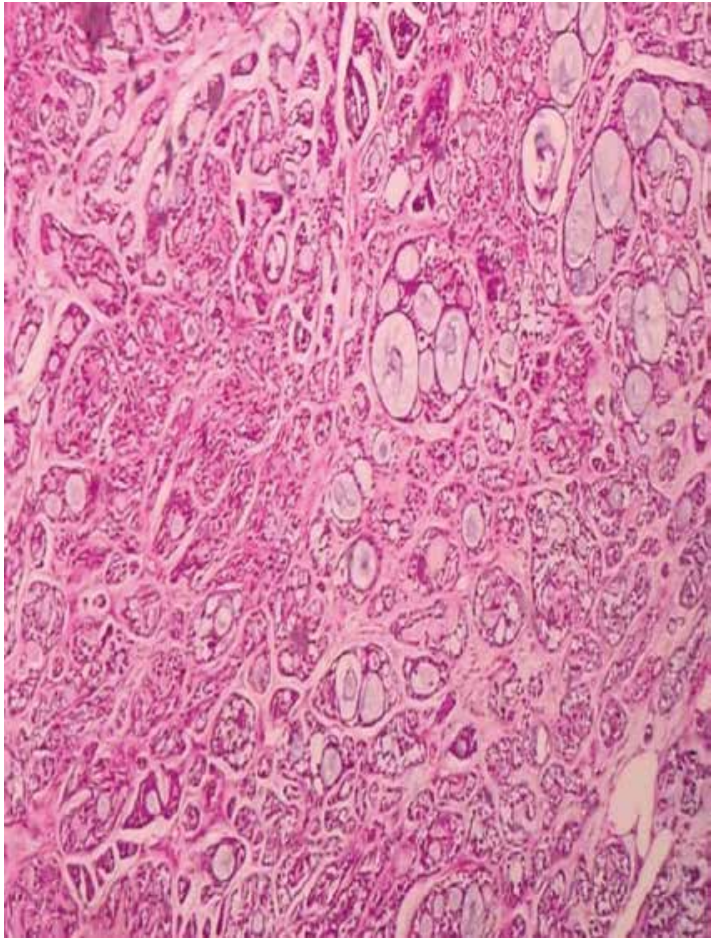

Figure 3. Pathological view of an adenoid cystic carcinoma

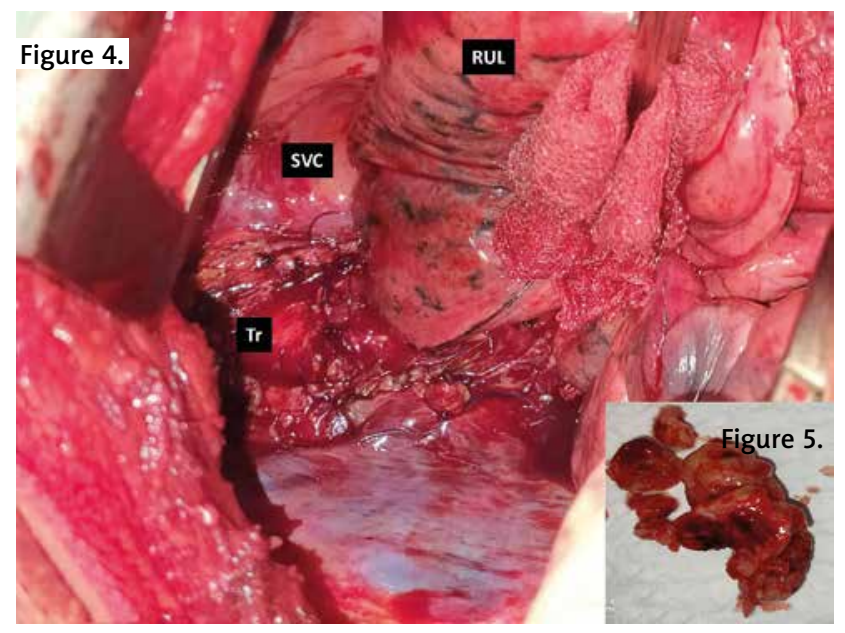

Figure 4. Preoperative view of posterior and lateral thoracotomy after ligation of azygos vein and tracheal resection and terminal anastomosis

Figure 5. En bloc resected tumor with friable parts SVC - superior vena cava, RUL - right upper lobe, $\mathrm{Tr}$ - trachea.

sis [1-4]. The challenge is where the tracheal obstruction is major and respiratory distress syndrome occurs. Preoperative endoscopic disobstruction can remove life-threatening asphyxia but the tumor spread and complete resection related prognosis become out of control. The 5 -year overall survival for tracheal ACC is approximately $30 \%$ with a reduction to less than $20 \%$ in cases of extratracheal extension. But with exclusive intraluminal disease, all the studies show a 5 -year overall survival rate of $50 \%[5,6]$. The main treatment of ACC consists of radical surgical removal with 
negative margins achievement and reconstruction [5-8]. Surgical techniques depend on the anatomic region. In cases of a standard resection in the distal trachea thoracotomy remain the optimal approach but sternotomy can be recommended when a carinal resection is probable; anastomosis is usually performed, though some practical limitations $[2,7]$. Postoperative radiotherapy when complete resection cannot be achieved ensures a positive impact on survival [8]. ACC of the trachea, in fact, proved to be radiosensitive. There is no accepted standard in the dosage of postoperative radiotherapy, but usually it is 60 Gy (2 Gy per fraction, five fractions per week, for a total of 6 weeks). Palliative endotracheal brachytherapy in advanced stages with intent can also be used. Our patient experienced excellent disease control, with a good quality of life, and prolonged overall survival by combining surgery and radiotherapy.

In conclusion, due to the rarity of the disease related clinical trials, there is not yet a standardized therapeutic approach to tracheal ACC. In our case disease control could be achieved by complete removal surgery and adjuvant radiotherapy. Surgery is often scheduled can be a life saving emergency.

\section{Disclosure}

The authors report no conflict of interest.

\section{References}

1. Sasson JP, Abdelrahman NG, Aquino S, Lev MH. Trachea: anatomy and pathology. In: Head and Neck Imaging. $4^{\text {th }}$ ed. Som PM, Curtin HD (eds.). Mosby St Louis, Missouri 2003; 1700-1726.

2. Yang PY, Liu MS, Chen CH, Lin CM, Tsao TCY. Adenoid cystic carcinoma of the trachea: a report of seven cases and literature review. Chang Gung Med J 2005; 28: 357-363.

3. Calzada AP, Miller M, Lai CK, Elashoff DA, Abemayor E, St John MA. Adenoid cystic carcinoma of the airway: a 30-year review at one institution. Am J Otolaryngol 2012; 33: 226-231.

4. Ahn Y, Chang H, Lim YS, Hah JH, Kwon TK, Sung MW, Kim KH. Primary tracheal tumors: review of 37 cases. J Thorac Oncol 2009; 4: 635-638.

5. Haresh KP, Prabhakar R, Rath GK, et al. Adenoid cystic carcinoma of the trachea treated with PET-CT based intensity modulated radiotherapy. J Thorac Oncol 2008; 3: 793-795.

6. Nuwal P, Dixit R, Singhal AK. Primary adenoid cystic carcinoma of trachea presenting as midline neck swelling and mimicking thyroid tumor: a case report and review of literature. Lung India 2010; 27: 167-169.

7. Choudhury BK, Barman G, Singh S, Ahmed K. Adenoid cystic carcinoma of the upper trachea: a rare neoplasm. J Clin Imaging Sci 2013; 3: 39-46.

8. Kwak SH, Lee KS, Chung MJ, Jeong YJ, kim GY, Kwon OJ. Adenoid cystic carcinoma of the airways: helical CT and histopathologic correlation. AJR Am J Roentgenol 2004; 183: 277-281. 